\title{
Corrigendum: Some class size conditions implying solvability of finite groups
}

\author{
[J. Group Theory 9 (2006) 787-797] \\ Antonio Beltrán and María José Felipe \\ (Communicated by Evgenii I. Khukhro)
}

The authors have realized that the proof of Step 1 in $[1$, Theorem $\mathrm{A}]$ is incomplete. Precisely, in the proof of Step 1 we suppose that there exists a $\pi$-element $x$ of index $m$ where $\pi$ is the set of primes dividing $m$ and we have the following situation: $C_{G}(x)$ is a direct product of a $p$-subgroup and a $p^{\prime}$-subgroup and the class sizes of such $p^{\prime}$-subgroup are at most two numbers: 1 or $n$. However, the case in which all of them are exactly equal to 1 , that is, when the $p^{\prime}$-subgroup is abelian, is omitted and this is the case we complete here by showing that it cannot happen.

Proof of Step 1 of Theorem A. Let $\pi$ be the set of primes dividing $m$ and suppose that there exists a $\pi$-element $x$ of index $m$. By using the primary decomposition of $x$ we may assume that $x$ is a $p$-element for some prime $p \in \pi$. Also, if $y$ is a $p^{\prime}$-element of $C_{G}(x)$, then $C_{G}(x y)=C_{G}(x) \cap C_{G}(y) \subseteq C_{G}(x)$ and thus $y$ has index 1 or $n$ in $C_{G}(x)$, which is a $p^{\prime}$-number. By Lemma $1, C_{G}(x)$ can be written as a direct product of a $p$-subgroup and a $p^{\prime}$-subgroup; so $C_{G}(x)=C_{G}(x)_{p} \times C_{G}(x)_{p^{\prime}}$. Moreover, the class sizes of $C_{G}(x)_{p^{\prime}}$ are 1 or $n$. If there are elements in this $p^{\prime}$-subgroup having both class sizes, then by applying [1, Theorems 4 and 7] we deduce that $G$ is solvable and the theorem is proved. We will prove that the other case leads to a contradiction. We assume then that $C_{G}(x)_{p^{\prime}}$ is abelian, whence we may write $C_{G}(x)=S_{x} \times T_{x}$, where $S_{x}$ is a $\pi$-subgroup and $T_{x}$ is an abelian $\pi$-complement of $G$. By a theorem of Wielandt (see for instance [2, Theorem 9.1.10]) all $\pi$-complements of $G$ are conjugate and every $\pi^{\prime}$-subgroup of $G$ lies in some $\pi$-complement of $G$. Also, we notice that every noncentral $\pi^{\prime}$-element of $G$ has index $m$ and that its centralizer is of the same type as of $x$. In fact, if $w$ is a noncentral $\pi^{\prime}$-element of $G$, then $w \in T_{x}^{g}$ for some $g \in G$. Thus $C_{G}\left(x^{g}\right)=S_{x}^{g} \times T_{x}^{g}=C_{G}(w)$.

This work is partially supported by Proyecto MTM2007-68010-C03-03 and by Proyecto GV-2009-021 and the first author is also supported by grant Fundació Caixa-Castelló P11B2008-09. 
Now, we claim that $n=|G|_{\pi^{\prime}} /|\mathbf{Z}(G)|_{\pi^{\prime}}$. Let $z$ be an element of index $m n$ and let us consider its decomposition $z=z_{\pi} z_{\pi^{\prime}}$. If $w=z_{\pi^{\prime}}$ is noncentral, by the above paragraph we have $C_{G}(w)=S_{w} \times T_{w}$, with $T_{w}$ an abelian $\pi$-complement of $G$. Then, $T_{w} \subseteq C_{G}(z)$, which is a contradiction. Hence, we can suppose $z$ to be a $\pi$-element. We prove that $C_{G}(z)=H_{z} \times K_{z}$, with $H_{z}$ a $\pi$-subgroup and $K_{z}$ an abelian $\pi^{\prime}$-subgroup. To see this, take any $\pi^{\prime}$-element $t \in C_{G}(z)$ and notice that $C_{G}(z t)=C_{G}(z) \cap C_{G}(t) \subseteq C_{G}(z)$. The maximality of the index of $z$ implies that $C_{G}(t) \cap C_{G}(z)=C_{G}(z)$, that is, $t \in \mathbf{Z}\left(C_{G}(z)\right)$, so $C_{G}(z)$ factorizes as wanted. Now, suppose that there exists a noncentral element $t \in K_{z}$. Then

$$
C_{G}(z) \subseteq C_{G}(t)=S_{t} \times T_{t}
$$

where $T_{t}$ is a $\pi$-complement of $G$, and thus, $z \in S_{t}$ and $T_{t} \subseteq C_{G}(z)$, which is a contradiction. Therefore, $K_{z}$ is central, and so the claim is proved.

Now, we take $\alpha$ an element of index $n$. By the equality obtained in the paragraph above, we can write $C_{G}(\alpha)=H_{\alpha} \times \mathbf{Z}(G)_{\pi^{\prime}}$ where $H_{\alpha}$ is some Hall $\pi$-subgroup of $G$. Then, we can assume that $\alpha$ is a $\pi$-element, lying in $\mathbf{Z}\left(H_{\alpha}\right)$. On the other hand, $H_{\alpha}$ contains some Sylow $p$-subgroup $P$ of $G$, so in particular, for the $p$-element $x$ fixed at the beginning of the proof, we have $x^{g} \in P \subseteq H_{\alpha}$ for some $g \in G$. It follows that $\alpha \in C_{G}\left(x^{g}\right)=S_{x}^{g} \times T_{x}^{g}$, and consequently $\alpha \in S_{x}^{g}$, so $T_{x}^{g} \subseteq C_{G}(\alpha)$ and this is the final contradiction.

\section{References}

[1] A. Beltrán, M. J. Felipe. Some class size conditions implying solvability of finite groups. J. Group Theory, 9 (2006) 787-797.

[2] D. J. S. Robinson. "A Course in the Theory of Groups". Graduate Texts in Mathematics 80. Second Edition. Springer-Verlag. Berlin-Heidelberg-New York 1995.

Received 1 September, 2010

Antonio Beltrán, Departamento de Matemáticas, Universidad Jaume I, 12071 Castellón, Spain

E-mail: abeltran@mat.uji.es

María José Felipe, Instituto Universitario de Matemática Pura y Aplicada, Universidad Politécnica de Valencia, 46022 Valencia, Spain

E-mail: mfelipe@mat.upv.es 